\title{
Hadron physics studies at KLOE
}

\author{
C. Bloise ${ }^{* \dagger}$ \\ Frascati Laboratory of INFN - Via E. Fermi, 40 - 00044 Frascati - Italy \\ E-mail: Caterina.Bloiseelnf.infn.it
}

The KLOE experiment at the Frascati $\phi$-factory has recently obtained results i) on the $\Gamma(\eta \rightarrow \gamma \gamma)$ partial width, with the study of $\eta$ production in $\gamma-\gamma$ interactions, and ii) on the form factors for Vector-meson to Pseudoscalars transitions, with the analysis of Dalitz decays $\phi \rightarrow P\left(\eta, \pi^{0}\right) \gamma^{*} \rightarrow$ $P e^{+} e^{-}$. The $\phi \rightarrow \eta e^{+} e^{-}$process has been analyzed using both, $\eta \rightarrow \pi^{+} \pi^{-} \pi^{0}$, and $\eta \rightarrow \pi^{0} \pi^{0} \pi^{0}$ decays. Pure samples, of about 13,000 and 30,000 events in the charged and neutral mode, respectively, with background contamination at the level of 2-3\%, have been obtained. The results completely supersede previous measurements, from the SND experiment, based on 213 events. We have also studied on a sample of 14,000 events, the $\phi \rightarrow \pi^{0} e^{+} e^{-}$decay for which no data on the transition form factor were available.

The $\gamma-\gamma$ couplings and the partial widths of light mesons can be measured at the $\phi$-factory through $e^{+} e^{-} \rightarrow e^{+} e^{-} \gamma^{*} \gamma^{*} \rightarrow e^{+} e^{-} X$ interactions. The KLOE analysis of $\eta$ production is based on an integrated luminosity of $240 \mathrm{pb}^{-1}$ of data, taken at the center of mass energy of $1 \mathrm{GeV}$, outside the $\phi$ peak to suppress backgrounds from $\phi$ decays, without any tag from $e^{+} / e^{-}$in the final state. The most precise measurement of the cross section for $\eta$ production, with two independent analyses selecting $\eta \rightarrow \pi^{+} \pi^{-} \pi^{0}$ and $\eta \rightarrow \pi^{0} \pi^{0} \pi^{0}$, has been used to obtain the $\Gamma(\eta \rightarrow \gamma \gamma)$ partial width at $5 \%$ precision level, $\Gamma(\eta \rightarrow \gamma \gamma)=\left(520 \pm 20_{\text {stat }} \pm 13_{\text {syst }}\right) \mathrm{eV}$.

The European Physical Society Conference on High Energy Physics -EPS-HEP2013

18-24 July 2013

Stockholm, Sweden

\footnotetext{
* Speaker.

$\dagger$ On behalf of the KLOE-2 Collaboration
} 


\section{Introduction}

The KLOE experiment at the Frascati $\phi$-factory took most of the data in 2004-2006, with $2.5 \mathrm{fb}^{-1}$ of integrated luminosity at the $\phi$ peak, and about $240 \mathrm{pb}^{-1}$ at $1 \mathrm{GeV}, 20 \mathrm{MeV}$ below the resonance, for the analysis of dipion, dilepton production and $\gamma-\gamma$ interactions. A new data taking campaign aiming to collect $5 \mathrm{fb}^{-1}$ in year 2014-15 is planned to extend the experimental program in kaon and hadron physics[1].

Recent results in hadronic physics are on the $\pi^{0}$ and $\eta$ coupling to photons. Meson to photon couplings and the transition form factors, TFFs, are fundamental measurements in hadron physics, of interest for the effective Lagrangians based on ChPT[2, 3] and for their extensions to the transition regime from soft, non-perturbative QCD, to hard interactions, described by $\mathrm{pQCD}$. The transition form factors for $\pi^{0}$ and $\eta$ in the time-like region are provided by the measurements of i) the Dalitz decays (e.g. $\eta \rightarrow \gamma e^{+} e^{-}$), ii) $V \rightarrow P \gamma^{*}$ transitions ( $\phi \rightarrow \eta e^{+} e^{-}, \phi \rightarrow \pi^{0} e^{+} e^{-}, \omega \rightarrow \pi^{0} e^{+} e^{-}$, ...), while iii) meson production in $\gamma-\gamma$ interactions $\left(e^{+} e^{-} \rightarrow e^{+} e^{-} \gamma^{*} \gamma^{*} \rightarrow e^{+} e^{-} \eta\left(\pi^{0}, \ldots\right)\right.$ ) gives the coupling to space-like photons, of interest also for the evaluation of light-by-light contribution to the anomalous magnetic moment of the muon[ [].

\section{2. $\eta$ production in $\gamma \gamma$ interactions}

The cross section $\sigma\left(e^{+} e^{-} \rightarrow e^{+} e^{-} \eta\right)$ is a convolution of the differential $\gamma \gamma$ luminosity and the $\gamma \gamma \rightarrow \eta$ cross section. The $\eta$ partial decay width $\Gamma(\eta \rightarrow \gamma \gamma)$ is obtained by extrapolating the value of $\sigma(\gamma \gamma \rightarrow \eta)$ from $\mathrm{q}^{2}$ space-like region to real photons.

The analysis[[]] is based on data collected with DAФNE operating off the $\phi$ peak, at $\sqrt{s}=1$ $\mathrm{GeV}$, to reduce the large background from $\phi$ decays. The $e^{+}$and $e^{-}$in the final state are mostly emitted in forward directions, outside the detector acceptance and they are not measured. The production of the $\eta$ meson is identified in two decay modes, $\eta \rightarrow \pi^{+} \pi^{-} \pi^{0}$ and $\eta \rightarrow \pi^{0} \pi^{0} \pi^{0}$. The charged and neutral channels are independently studied, exploiting the performance of the KLOE tracking system, and the calorimeter, respectively. The most relevant background comes from $e^{+} e^{-} \rightarrow \eta \gamma$ and, for both measurements, the yield of $\eta$ mesons is controlled by the $e^{+} e^{-} \rightarrow \eta \gamma$ cross section, measured in the same data sample. Data are pre-selected with a background-rejection filter [6] requiring at least two neutral clusters in the calorimeter coming from the interaction region (IP), and with total energy $100 \mathrm{MeV}<E_{\text {tot }}<900 \mathrm{MeV}$, to reject low energy background events and the high-rate processes $e^{+} e^{-} \rightarrow e^{+} e^{-}(\gamma), e^{+} e^{-} \rightarrow \gamma \gamma$. A 1/20 sample of unfiltered data is also reconstructed to evaluate the efficiency of the filter procedure.

Candidates for $\eta \rightarrow \pi^{+} \pi^{-} \pi^{0}$ have $\mathrm{i}_{c h}$ ) only two neutral, prompt clusters; $\mathrm{ii}_{c h}$ ) at least two tracks from the IP with opposite curvature and $\left|\mathbf{p}_{1}\right|+\left|\mathbf{p}_{2}\right|<700 \mathrm{MeV}$, while for the $\eta \rightarrow \pi^{0} \pi^{0} \pi^{0}$ channel $\mathrm{i}_{n}$ ) six neutral prompt clusters with $E_{\gamma}>15 \mathrm{MeV}$ and polar angle $23^{\circ}<\theta_{\gamma}<157^{\circ}$, and ii $_{n}$ ) no tracks in the drift chamber have been required.

The correlation of the squared missing mass, $m_{m i s}^{2}$, and the $\eta$ momentum components, $p_{L \eta}$ and $p_{T \eta}$, longitudinal and transverse to the beam axis, are used to separate signal from background. Many background contributions are considered: i) the $e^{+} e^{-} \rightarrow \eta \gamma$ process is a source of irreducible background when $\eta$ decays to $3 \pi$ and the monochromatic photon, $E_{\gamma}=350 \mathrm{MeV}$, is emitted at small polar angles remaining undetected; ii) the $e^{+} e^{-} \rightarrow \omega \pi^{0}$ processes whose cross section has 

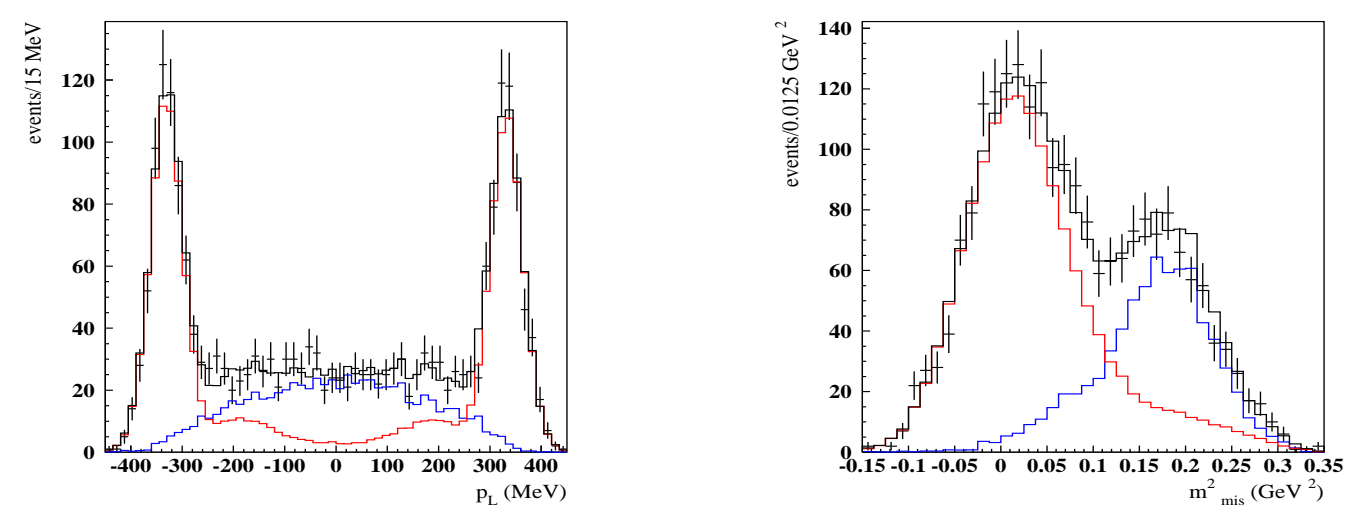

Figure 1: Projections of the 2-dimensional fit of the selected sample of $e^{+} e^{-} \rightarrow e^{+} e^{-} \eta$ with $\eta \rightarrow 3 \pi^{0}$. Left: distribution of the $6 \gamma$ longitudinal momentum. Right: distribution of the squared missing mass. The contribution of the signal is blue, $e^{+} e^{-} \rightarrow \eta \gamma$ is red.

been measured with data from the same runs [7] for a precise evaluation of their contribution; iii) the $e^{+} e^{-} \rightarrow K_{L} K_{S}$; iv) the $e^{+} e^{-} \rightarrow K^{+} K^{-}$(for the charged channel only); v) Bhabha radiative events, $e^{+} e^{-} \rightarrow e^{+} e^{-} \gamma$ that, given the large cross section, are a background source for the charged channel in case of accidental or split clusters. Bhabha-scattering events are partially rejected exploiting the $\pi^{ \pm}$vs $e^{ \pm}(\gamma)$ separation capability of the calorimeter, mostly based on the precision measurement of the time-of-flight. For the neutral channel only, the background from vi) the $e^{+} e^{-} \rightarrow a_{0}(980) \gamma \rightarrow$ $\eta \pi^{0} \gamma$; vii) $e^{+} e^{-} \rightarrow f_{0}(980) \gamma \rightarrow \pi^{0} \pi^{0} \gamma$ and viii) $e^{+} e^{-} \rightarrow \eta^{\prime} \gamma$, when $\eta^{\prime}$ decays to neutrals, has been also studied.

A kinematic fit constrained by the invariant mass of the three-body final state (equal to the $\eta$ mass) has been applied on both analyses, rejecting events with poor fit quality.

The number of signal events is derived from a bi-dimensional fit to data, together with the number of all of the background components, that are in agreement with expectations from cross sections and MonteCarlo-evaluated efficiencies. The variables used to discriminate the signal are the squared missing mass and the $\eta$ transverse momentum, in the interval $-0.15 \mathrm{GeV}^{2}<m_{\text {mis }}^{2}<$ $0.25 \mathrm{GeV}^{2}$ and $p_{T \eta}<300 \mathrm{MeV}$ (2720 events), in the case of charged decays $\eta \rightarrow \pi^{+} \pi^{-} \pi^{0}$, and the squared missing mass and the $\eta$ longitudinal momentum in the interval $-0.15 \mathrm{GeV}^{2}<m_{\text {mis }}^{2}<$ $0.35 \mathrm{GeV}^{2}$ and $-450 \mathrm{MeV}<p_{L \eta}<450 \mathrm{MeV}$ (2166 events), for the neutral sample of $\eta \rightarrow \pi^{0} \pi^{0} \pi^{0}$. For the latter, all backgrounds except $\eta \gamma$ are negligible and we fit data with two components only, obtaining $723 \pm 32$ signal events, shown in Fig.1. The projections of the bi-dimensional plot, the $m_{m i s}^{2} \times p_{T \eta}$, for data and all of the background components in the charged sample are shown in Figure 2 . In this case the fit returns $394 \pm 29$ signal events.

The systematics are evaluated varying all of the analysis cuts, obtaining a relative error of $2.5 \%$.

The results are: $\sigma\left(e^{+} e^{-} \rightarrow e^{+} e^{-} \eta \rightarrow e^{+} e^{-} \pi^{+} \pi^{-} \pi^{0}\right)=\left(7.84 \pm 0.57_{\text {stat }} \pm 0.23_{\text {syst }} \pm 0.16_{\mathrm{FF}}\right) \mathrm{pb}$, and $\sigma\left(e^{+} e^{-} \rightarrow e^{+} e^{-} \eta \rightarrow e^{+} e^{-} 3 \pi^{0}\right)=\left(10.43 \pm 0.48_{\text {stat }} \pm 0.29_{\text {syst }} \pm 0.07_{\mathrm{FF}}\right) \mathrm{pb}$. Using the $\eta$ branching fractions from Ref.[8]:

$$
\sigma\left(e^{+} e^{-} \rightarrow e^{+} e^{-} \eta\right)=\left(34.5 \pm 2.5_{\mathrm{stat}} \pm 1.0_{\mathrm{syst}} \pm 0.7_{\mathrm{FF}} \pm 0.4_{\mathrm{BR}}\right) \mathrm{pb}
$$



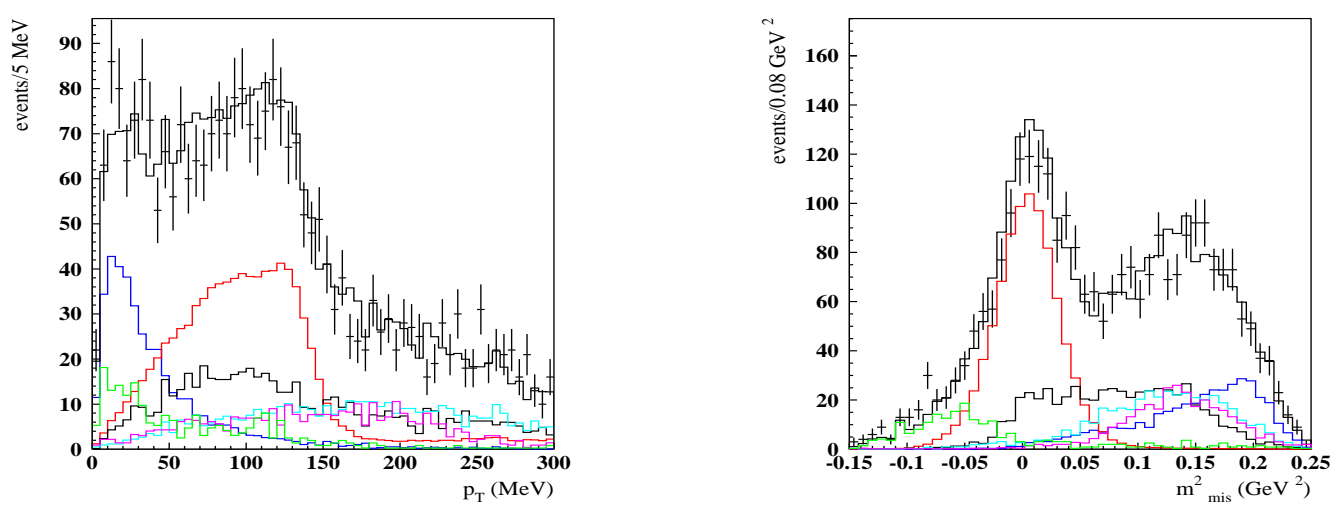

Figure 2: Projections of the 2-dimensional fit of the selected sample of $e^{+} e^{-} \rightarrow e^{+} e^{-} \eta$ with $\eta \rightarrow \pi^{+} \pi^{-} \pi^{0}$. Left: distribution of the transverse momentum of the $\pi^{+} \pi^{-} \gamma \gamma$ system. Right: distribution of the squared missing mass. The contribution of the signal is blue, $e^{+} e^{-} \rightarrow \eta \gamma$ is red, $e^{+} e^{-} \rightarrow \omega \pi^{0}$ is black, $e^{+} e^{-} \rightarrow$ $e^{+} e^{-} \gamma$ is green, $e^{+} e^{-} \rightarrow K^{+} K^{-}$is light blue and $e^{+} e^{-} \rightarrow K_{S} K_{L}$ is purple.

and

$$
\sigma\left(e^{+} e^{-} \rightarrow e^{+} e^{-} \eta\right)=\left(32.0 \pm 1.5_{\mathrm{stat}} \pm 0.9_{\mathrm{syst}} \pm 0.2_{\mathrm{FF}} \pm 0.2_{\mathrm{BR}}\right) \mathrm{pb}
$$

are obtained from the analysis of the charged, and the neutral $\eta$ decay channel, respectively.

The partial width of the $\eta$ meson, $\Gamma(\eta \rightarrow \gamma \gamma)$, is calculated from the $\gamma \gamma$ differential luminosity of Ref.[9], taking into account the $q_{1}$ and $q_{2} 4$-momenta distributions for each of the decay modes,

$$
\sigma\left(e^{+} e^{-} \rightarrow e^{+} e^{-} X\right)=\frac{8 \pi^{2}}{m_{X}} \Gamma_{X \rightarrow \gamma \gamma} \int\left|F\left(q_{1}^{2}, q_{2}^{2}\right)\right|^{2} \delta\left(w^{2}-m_{X}^{2}\right) \Phi\left(q_{1}, q_{2}\right) \frac{d \mathbf{q}_{1}}{E_{1}} \frac{d \mathbf{q}_{2}}{E_{2}}
$$

where the $\gamma \gamma$ differential luminosity is $\Phi\left(q_{1}, q_{2}\right), \Gamma_{X \rightarrow \gamma \gamma}$ is the radiative width, $w^{2}=\left(q_{1}+q_{2}\right)^{2}$, and $F\left(q_{1}^{2}, q_{2}^{2}\right)$ is the transition form factor.

The theoretical error in evaluating $\sigma(\gamma \gamma \rightarrow \eta)$ has been added to the systematic error, including the uncertainty on the form factor.

$$
\begin{gathered}
\eta \rightarrow \pi^{+} \pi^{-} \pi^{0} \quad \Gamma(\eta \rightarrow \gamma \gamma)=\left(548 \pm 40_{\mathrm{stat}} \pm 16_{\mathrm{syst}} \pm 14_{\mathrm{FF}} \pm 7_{\mathrm{BR}}\right) \mathrm{eV} \\
\rightarrow \pi^{0} \pi^{0} \pi^{0} \quad \Gamma(\eta \rightarrow \gamma \gamma)=\left(509 \pm 23_{\mathrm{stat}} \pm 14_{\mathrm{syst}} \pm 8_{\mathrm{FF}} \pm 4_{\mathrm{BR}}\right) \mathrm{eV}
\end{gathered}
$$

The two measurements are combined accounting for their correlations to obtain the most precise result from a single experiment: $\Gamma(\eta \rightarrow \gamma \gamma)=\left(520 \pm 20_{\text {stat }} \pm 13_{\text {syst }}\right) \mathrm{eV}$, in agreement with the world average of Ref.[8].

3. $\phi \rightarrow \pi^{0} e^{+} e^{-}$

The $\phi \rightarrow \pi^{0} e^{+} e^{-}$decays are indentified from the reconstruction of i) two clusters in the calorimeter, consistent with $\pi^{0}$ decay products, and ii) two tracks of opposite charge in the drift chamber (DC). Both, the clusters, and the tracks, are originated at the IP. The $e^{+} e^{-}$pairs are identified from time-of-flight precision measurement of their clusters in the calorimeter. The dominant background contribution comes from Bhabha scattering processes, overwhelming the signal 

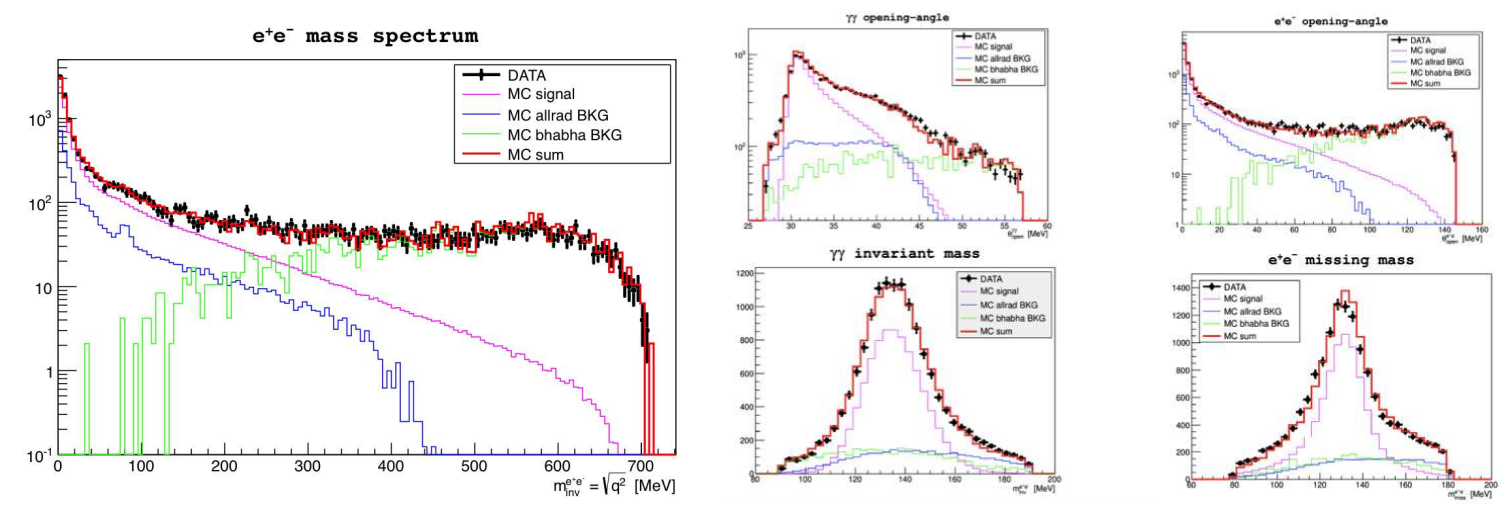

Figure 3: Invariant mass $\left(\mathrm{M}_{e e}\right)$ distribution of $\phi \rightarrow e^{+} e^{-} \pi^{0}$ events. Left: Selected sample of $\sim 14,000$ candidates, including contamination from Bhabha-scattering processes and $\phi \rightarrow \pi^{0} \gamma$ decays. Right: DataMonteCarlo comparison on several distributions: $\gamma-\gamma$ opening angle, $e^{+} e^{-}$opening angle, $\gamma-\gamma$ invariant mass, missing invariant mass reconstructed from beam-beam and $e^{+} e^{-} 4$-momenta.

at $M_{e e}>0.3 \mathrm{GeV}$ (Fig. B, left). The other relevant contribution is from $\phi \rightarrow \pi^{0} \gamma$ decays. We suppress the part due to photon conversions to $e^{+} e^{-}$pairs on the beam pipe (BP) or DC walls by proper cuts on the track distance and invariant mass, measured at the BP and DC walls.

A sample of $\sim 14,000$ candidates is obtained from the analysis of $1.7 \mathrm{fb}^{-1}$ of integrated luminosity, with total efficiency of about $13 \%$ at low invariant masses, $\mathrm{M}_{e e}<0.2 \mathrm{GeV}$. Studies are in progress for the evaluation of the accuracy on i) the background contamination and ii) the analysis efficiencies. Fig.3 (right) shows the data-MonteCarlo comparison on several distributions for all of the contributing terms to the selected sample.

4. $\phi \rightarrow \eta e^{+} e^{-}$

The Dalitz $\phi$ decay $\phi \rightarrow \eta e^{+} e^{-}$has been independently studied selecting both, $\eta \rightarrow \pi^{+} \pi^{-} \pi^{0}$, and $\eta \rightarrow \pi^{0} \pi^{0} \pi^{0}$ decay modes, on a sample of about $1.7 \mathrm{fb}^{-1}$ of integrated luminosity. The $e^{+} e^{-}$ invariant-mass distributions have been obtained on the basis of 13,000 and 30,000 events selected in the charged, and the neutral decay mode, respectively.

For charged $\eta$ decays, four tracks and two neutral calorimeter clusters coming from the IP are required, while for the neutral $\eta$ decay mode, events with two tracks and six neutral clusters from the IP are selected. The final state must have an invariant mass consistent with the $\eta$ mass. The $e^{+}$ $e^{-}$pairs are identified from the time-of-flight measurement, as in Sec. 3 .

The missing invariant mass reconstructed from beam-beam and $e^{+} e^{-}$4-momenta, $\mathrm{M}_{\text {recoil-ee }}$, is used to identify $\phi \rightarrow \eta e^{+} e^{-}$candidates, $536.5<M_{\text {recoil-ee }}<554.5 \mathrm{MeV}$. The contamination from $\phi \rightarrow \eta \gamma$ decays, mainly due to events with photon conversion to an $e^{+} e^{-}$pair on the beam pipe (BP) or DC walls, is suppressed tracing back $e^{+} / e^{-}$candidates and reconstructing their invariant mass $\left(M_{e e}\right)$ and their distance $\left(D_{e e}\right)$ both, at the BP, and DC wall surfaces. Both variables are small in case of photon conversion, and are rejected with: $\left[M_{e e}(B P)<10 \mathrm{MeV}\right.$ and $D_{e e}(B P)<2 \mathrm{~cm}$ ] or $\left[M_{e e}(D C)<120 \mathrm{MeV}\right.$ and $D_{e e}(D C)<4 \mathrm{~cm}$ ]. An additional cut, on the recoil mass to the $e^{+} e^{-} \pi^{+} \pi^{-}$system, which must be consistent with $\pi^{0}$ mass, $100<M_{\text {recoil-(ee } \pi \pi)}<160 \mathrm{MeV}$, is used in the analysis of the charged channel $\eta \rightarrow \pi^{+} \pi^{-} \pi^{0}$. 

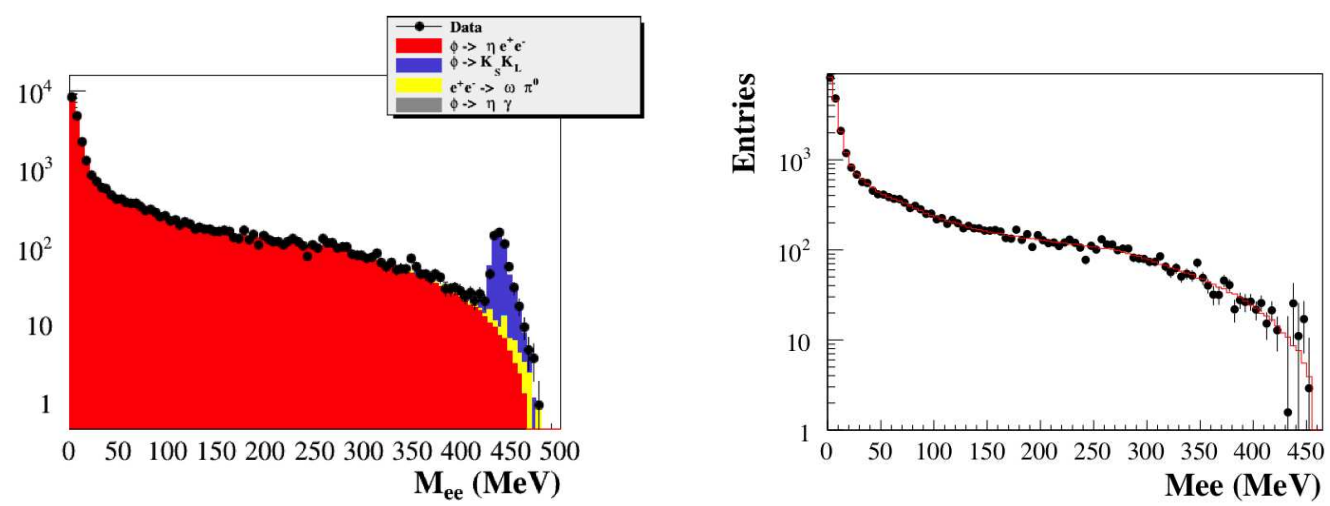

Figure 4: Invariant mass $\left(\mathrm{M}_{e e}\right)$ distribution of $\phi \rightarrow e^{+} e^{-} \eta$ events. Left: Selected sample of 30,577 candidates with $\eta \rightarrow \pi^{0} \pi^{0} \pi^{0}$ decays, including $\sim 3 \%$ background contamination. Right: $M_{e e}$ distribution after background subtraction (black points) with results from the fit (red histogram).

At the end of the analysis chain we obtain consistent distributions from both samples, on the basis of 30,577 events selected in the neutral mode, with $\sim 3 \%$ background, and 13,254 in the charged decay channel, with $\sim 2 \%$ contamination.

The form factor has been obtained by a fit to the $\mathrm{M}_{e e}$ distribution using Eq.4.1:

$\frac{d \Gamma\left(\phi \rightarrow e^{+} e^{-} \eta\right)}{d M_{e e}} \propto \Gamma(\phi \rightarrow \eta \gamma) \frac{\left|F_{\phi \eta}\left(M_{e e}^{2}\right)\right|^{2}}{M_{e e}} \sqrt{1-\frac{4 m^{2}}{M_{e e}^{2}}}\left(1+\frac{2 m^{2}}{M_{e e}^{2}}\right)\left[\left(1+\frac{M_{e e}^{2}}{m_{\phi}^{2}-m_{\eta}^{2}}\right)^{2}-\frac{4 m_{\phi}^{2} M_{e e}^{2}}{\left(m_{\phi}^{2}-m_{\eta}^{2}\right)^{2}}\right]^{\frac{3}{2}}$

with

$$
\left|F_{\phi \eta}\left(M_{e e}^{2}\right)\right|=\frac{1}{1-b_{\phi \eta} M_{e e}^{2}}
$$

and taking into account residual background. Fig. $\theta$ refers to $\phi \rightarrow \eta e^{+} e^{-}, \eta \rightarrow 3 \pi^{0}$ analysis. Data are black points and the fit results, giving $b_{\phi \eta}=\left(1.17 \pm 0.10_{-0.08}^{+0.09}\right) \mathrm{GeV}^{-2}$, are superimposed (red histogram). We have also obtained the branching fraction, $B R\left(\phi \rightarrow \eta e^{+} e^{-}\right)=(1.131 \pm$ $\left.0.007_{-0.006}^{+0.011} \pm 0.031_{\text {norm }}\right) \times 10^{-4}$, where the second term is the statistical error, the third is the evaluation of systematics, the fourth is the uncertainty on the normalization to the total cross section, $\sigma\left(e^{+} e^{-} \rightarrow \phi\right) \cdot B R\left(\eta \rightarrow 3 \pi^{0}\right)$. The results completely superseded previous measurements[10] and are in agreement with expectations from the VMD model[11].

\section{Conclusions}

Several results on meson transition form factors and $\gamma-\gamma$ physics have been recently finalized on the basis of KLOE data, selected from the $2.5 \mathrm{fb}^{-1}$ of integrated luminosity at the $\phi$ peak, and $240 \mathrm{pb}^{-1}$ of off-peak data, at $1 \mathrm{GeV}$. We have obtained the most precise measurements of i) the transition form factor, $F_{\phi \eta}$, with the analysis of $\phi \rightarrow e^{+} e^{-} \eta$ decays, and ii) the radiative $\eta$ width, $\Gamma(\eta \rightarrow \gamma \gamma)=\left(520 \pm 20_{\text {stat }} \pm 13_{\text {syst }}\right) \mathrm{eV}$, from $\eta$ production in $\gamma-\gamma$ interactions, $e^{+} e^{-} \rightarrow e^{+} e^{-} \eta$.

In 2013 the KLOE detector has been upgraded i) to improve vertex reconstruction near the beam interaction region, ii) to increase the acceptance for low polar angle photons, and iii) to reconstruct particles passing through the DAФNE final focusing region. A cylindrical tracking 
chamber based on the Gaseous Electron Multiplier (GEM) technology[12] has been installed between the beam pipe and the big Drift Chamber to track particles closer to their origin; two small stations of LYSO calorimeters [13] have been placed on the beam pipe for the detection of low polar angle photons; the final focusing region has been instrumented with sampling calorimeters done by five layers of tungsten interleaved with scintillator tiles coupled to fibers that are readout on one side by silicon photomultipliers[14].

Two different kinds of $\gamma-\gamma$ taggers have been installed for the detection of $e^{+} / e^{-}$from $e^{+} e^{-} \rightarrow$ $e^{+} e^{-} X$, crucial for the identification of such processes while running at the $\phi$ resonance. The two $\left(e^{+}\right.$and $\left.e^{-}\right)$stations for the detection of particles in the 160-230 MeV energy range are constituted by LYSO calorimeters [15] installed $1.5 \mathrm{~m}$ from the IP; the other two are scintillator strips [16] installed $11 \mathrm{~m}$ from the IP, in a Roman pot placed on the $D A \Phi N E$ first bending dipole, to detect electrons with $\mathrm{E}_{e^{ \pm}}>400 \mathrm{MeV}$.

Most of the physics program with the detector upgrades for the new run at $D A \Phi N E$ is discussed in Ref.[1]. In particular, with the forthcoming data taking in year 2014-2015 and the operation of the tagger stations we expect to obtain, among other measurements, the $\Gamma\left(\pi^{0} \rightarrow \gamma \gamma\right)$ width at $1 \%$ precision level, and the transition form factors at low $\mathrm{q}^{2}$ with 5-6\% per-bin accuracy[17].

\section{References}

[1] G. Amelino-Camelia, F. Archilli, D. Babusci et al., EPJ C68, 619 (2010)

[2] C. Terschlusen, S. Leupold, Prog.Part.Nucl.Phys. 67, 401 (2012), 1111.4907

[3] S.P. Schneider, B. Kubis, F. Niecknig, Phys.Rev. D86, 054013 (2012), 1206.3098

[4] F. Jegerlehner, A. Nyffeler, Phys.Rept. 477, 1 (2009)

[5] D. Babusci et al. (KLOE-2), JHEP 1301, 119 (2013), 1211 . 1845

[6] F. Ambrosino et al., Nucl.Instrum.Meth. A534, 403 (2004), phys ics / 0404100

[7] F. Ambrosino et al. (KLOE collaboration), Phys.Lett. B669, 223 (2008), 0807 . 4909

[8] J. Beringer et al. (Particle Data Group), Phys.Rev. D86, 010001 (2012)

[9] F. Nguyen, F. Piccinini, A. Polosa, Eur.Phys.J. C47, 65 (2006), hep-ph / 0602205

[10] M.N. Achasov et al., Phys. Lett. B504, 275 (2001)

[11] L.G. Landsberg, Phys. Rept. 128, 301 (1985)

[12] A. Balla, G. Bencivenni, P. Branchini et al., Acta Phys.Polon.Supp. 6(4), 1053 (2013)

[13] F. Happacher, M. Martini, S. Miscetti, I. Sarra, Nucl.Phys.Proc.Suppl. 197, 215 (2009), 0906.1157

[14] M. Cordelli, G. Corradi, F. Happacher et al., Nucl.Instrum.Meth. A617, 105 (2010)

[15] D. Babusci, C. Bini, P. Ciambrone et al., Nucl.Instrum.Meth. A617, 81 (2010), 0906.0875

[16] F. Archilli, D. Babusci et al., Nucl.Instrum.Meth. A617, 266 (2010)

[17] D. Babusci, H. Czyz, F. Gonnella, S. Ivashyn, M. Mascolo et al., Eur.Phys.J. C72, 1917 (2012) 